\section{OPEN ACCESS}

Edited by:

Alessandro Isidori,

Hematology and Stem Cell Transplant Center, AORMN Hospital, Italy

Reviewed by:

Ying-Jun Chang

Peking University People's

Hospital, China

Michele Malagola,

University of Brescia, Italy

*Correspondence:

Dolores Grosso

dolores.grosso@jefferson.edu

Specialty section

This article was submitted to

Hematologic Malignancies,

a section of the journal

Frontiers in Oncology

Received: 24 July 2019

Accepted: 27 August 2019

Published: 17 September 2019

Citation:

Grosso D, Leiby B, Carabasi M,

Filicko-O'Hara J, Gaballa S,

O'Hara W, Wagner JL and

Flomenberg N (2019) The Presence of

a CMV Immunodominant Allele in the

Recipient is Associated With

Increased Survival in CMV Positive

Patients Undergoing Haploidentical

Hematopoietic Stem Cell

Transplantation. Front. Oncol. 9:888.

doi: 10.3389/fonc.2019.00888

\title{
The Presence of a CMV
} Immunodominant Allele in the Recipient Is Associated With Increased Survival in CMV Positive Patients Undergoing Haploidentical Hematopoietic Stem Cell Transplantation

Dolores Grosso ${ }^{1 *}$, Benjamin Leiby ${ }^{2}$, Matthew Carabasi ${ }^{1}$, Joanne Filicko-O'Hara ${ }^{1}$,
Sameh Gaballa ${ }^{1}$, William O'Hara ${ }^{1}$, John L. Wagner ${ }^{1}$ and Neal Flomenberg

${ }^{1}$ Blood and Marrow Transplant Program, The Sidney Kimmel Cancer Center at Thomas Jefferson University, Philadelphia, PA, United States, ${ }^{2}$ Pharmacology and Experimental Therapeutics, The Sidney Kimmel Cancer Center at Thomas Jefferson University, Philadelphia, PA, United States

Specific major histocompatibility (MHC) class I alleles dominate anti-CMV responses in a hierarchal manner. These CMV immunodominant (IMD) alleles are associated with a higher magnitude and frequency of cytotoxic lymphocyte responses as compared to other human leukocyte antigen (HLA) alleles. CMV reactivation has been associated with an increased incidence of graft-vs.-host disease and non-relapse mortality, as well as protection from relapse in HLA-matched HSCT settings. Less is known about the impact of CMV reactivation on these major outcomes after haploidentical $(\mathrm{HI})$ HSCT, an increasingly applied therapeutic option. In HI HSCT, the efficiency of the immune response is decreased due to the immune suppression required to cross the $\mathrm{MHC}$ barrier as well as $\mathrm{MHC}$ mismatch between presenting and responding cells. We hypothesized that the presence of a CMV IMD allele would increase the efficiency of CMV responses after HI HSCT potentially impacting CMV-related outcomes. In this retrospective, multivariable review of $216 \mathrm{HI}$ HSCT patients, we found that CMV+ recipients possessing at least 1 of 5 identified CMV IMD alleles had a lower hazard of death (HR $=0.40, p=0.003)$ compared to $C M V+$ recipients not possessing a CMV IMD allele, and an overall survival rate similar to their CMV- counterparts. The analysis delineated subgroups within the $\mathrm{CMV}+$ population at greater risk for death due to $\mathrm{CMV}$ reactivation.

Keywords: CMV, immunodominant allele, haploidentical, transplant outcomes, graft versus host disease, DNA terminase complex inhibitor

\section{INTRODUCTION}

CMV reactivation has been associated with protection from relapse in human leukocyte antigen (HLA) matched related and unrelated hematopoietic stem cell transplant (HSCT) settings (1-3), most pronounced in patients with acute myeloid leukemia (AML) (4). However, in many series, benefits derived from CMV reactivation in terms of relapse are outweighed by accompanying 
increases in non-relapse mortality (NRM) $(1,5)$ and death $(5,6)$. Herpesviruses such as CMV share peptide sequence homology with humans resulting in cross-reactive cytotoxic lymphocyte (CTL) responses to both the virus and self, providing an explanation as to why CMV has been associated with graft vs. host disease (GVHD) and protection from relapse (7-9).

After haploidentical HSCT (HI HSCT), CMV reactivation rates are higher than typically seen following HLA matched HSCT (10-13), in part due to immune suppression of donor effector cells necessary to safely cross the major histocompatibility complex (MHC) barrier (14). However, HI HSCT also represents a situation where CMV epitope presentation to responder cells occurs in the context of MHC mismatch, which may further decrease the efficacy of the immune response to CMV (15).

Robust reconstitution of CMV-specific CTLs (16), adoptive transfer of CMV-specific $\mathrm{T}$ cells (17), and aggressive pharmacological approaches to CMV prophylaxis (18) have been shown to reduce CMV reactivation and disease post HI HSCT. However, there is limited information regarding the impact of CMV reactivation on outcomes such as relapse incidence and overall survival (OS) after HI HSCT. In a small analysis of 36 HI HSCT recipients, Lin et al. (19) identified a protective effect of CMV reactivation on relapse in AML subjects which was offset by increased NRM. In HI HSCT regimens using cyclophosphamide (CY) tolerization as GVHD prophylaxis, CMV reactivation was associated with increased NRM in one analysis (20) but a causal relationship between CMV reactivation and any major transplant outcome was not found in two others $(12,21,22)$. Thus, despite a high reactivation rate after HI HSCT, a clear pattern of relapse protection or survival differences due to CMV has not emerged at least in transplants using CY tolerization approaches.

Multiple MHC alleles are capable of presenting CMV antigens to CTLs (23), however a hierarchal functionality exists (24-28) such that specific MHC class I alleles dominate CMV responses preferentially presenting CMV epitopes even in the presence of other HLA alleles capable of doing so.

HLA A*01:01 $(25,29), A^{*} 02: 01(24,26,28), B^{*} 07: 02(24,28$, $30,31), B^{*} 08: 01(28,32)$, and $C^{*} 07: 02(27,33)$ are amongst the most immunodominant (IMD) CMV alleles presenting pp65, immediate early 1 and pp50 CMV epitopes which have been identified as the most immunogenic of CMV proteins $(28,34-$ 36). In addition to hierarchal dominance, these alleles have been associated with eliciting a higher response frequency of CMV-specific CTLs (24-26, 29, 37), a greater magnitude of CTL expansion upon stimulation $(24,26)$, and a more stable and stronger binding affinity with responding CMV-specific CTLs $(30,32)$.

Because CMV IMD alleles are associated with greater efficiency and CTL frequencies in CMV responses, we previously hypothesized that in HI HSCT, the expression of a CMV IMD allele by the donor, the recipient, or a match of a CMV IMD allele on the shared haplotype could strengthen anti-CMV responses in patients in which these alleles were present. Examination of CMV-related outcomes such as relapse, NRM, and OS based on the presence or absence of CMV IMD alleles would potentially help delineate recipient CMV risks and clarify the effects of CMV reactivation in HI HSCT.

A preliminary univariate analysis performed at our institution (38) showed that CMV reactivation rates and CMV copy number were not significantly influenced post HI HSCT by the presence or the matching of CMV IMD alleles. However, the expression of one or more CMV IMD alleles in the recipient only was associated with lower rates of NRM and relapse, as well as higher OS as compared to recipients without a CMV IMD allele.

\section{MATERIALS AND METHODS}

The objective of this single institution, retrospective, multivariable analysis was to confirm a beneficial impact of recipient CMV IMD allele positivity (CMV IMD+) on outcomes post HI HSCT.

There were three testing groups. The primary group consisted of $\mathrm{CMV}$ positive $(\mathrm{CMV}+)$ recipients expressing one or more CMV IMD alleles. The outcomes of this group were compared to both $\mathrm{CMV}+$ recipients not possessing a CMV IMD allele and $\mathrm{CMV}$ negative (CMV-) recipients, none of whom had evidence of a primary CMV infection after HI HSCT. Comparison to the second group allowed an analysis of the impact of CMV IMD alleles in the setting of CMV reactivation which occurred in the majority of $\mathrm{CMV}+$ patients. $\mathrm{CMV}$ - recipients were also used as a comparator group to confirm that the effects of CMV IMD alleles were specific to the setting of CMV reactivation.

All patients underwent HI HSCT on any of the Institutional Review Board-approved two-step research studies at Thomas Jefferson University Hospital between 2006 and 2019. The two step approach is shown in Figure 1. In this regimen, fixed dosing of $\mathrm{CD}^{+}$cells and a consistent GVHD prophylaxis approach provides a consistent platform to compare treatment effects amongst groups. Written consent was obtained from all patients in accordance with the Declaration of Helsinki.

HSCT outcomes examined for these groups were relapse, incidences of acute (aGVHD) and chronic (cGVHD), NRM, and OS. Confounders considered in the analysis were patient and donor age, paternal recipient based on a consistent finding of better outcomes in this patient group at our institution (39), conditioning intensity, myeloid vs. other diagnoses, Revised Disease Risk Index (RDRI) (40), CMV reactivation in the first 100 days post HSCT, Hematopoietic Cell Transplantation-Specific Comorbidity Index (41), the use of steroids within the first 100 days of HSCT, the presence of a killer immunoglobulinlike receptor (KIR) mismatch as defined by Ruggeri et al. (42), and donor B haplotype (43). Race/ethnicity (Race) was also considered as a confounder in the model to exclude the possibility that CMV IMD alleles, most commonly associated with Caucasians of European ancestry, were acting as a surrogate for the effect of Caucasian race on outcomes. Race has been found to have significant impacts on post HSCT outcomes in some analyses $(44,45)$.

To be included in the analysis, patients had to have had successful donor engraftment and no evidence of disease on the day +28 post marrow studies. Every patient transplanted 


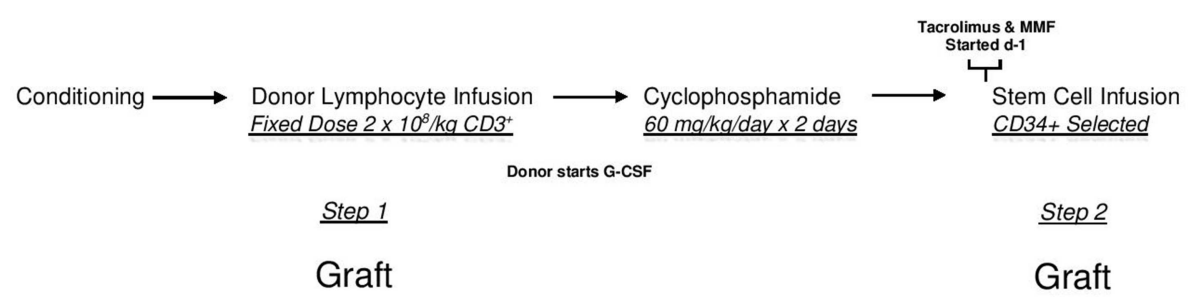

FIGURE 1 | The two step approach. After conditioning all patients receive an unmanipulated donor product containing a fixed dose of $2 \times 10^{8} / \mathrm{kg}$ donor $\mathrm{CD} 3^{+}$cells (DLI-step 1 of transplant). Two days after the DLI, Cyclophosphamide is infused at a dose of $60 \mathrm{mg} / \mathrm{kg} /$ day $\times 2$ days for bidirectional tolerization of lymphocytes. After a day of rest, a CD34 selected donor stem cell product is infused (step 2 of transplant). Conditioning regimens were myeloablative consisting of 12 Gy total body irradiation ( $n=128)$; reduced intensity total of 78 patients consisting of Fludarabine, 2 Gy total body irradiation, plus Cytarabine $(n=13)$ or Thiotepa $(n=45)$ or Busulfan ( $n=20)$; or non-myeloablative (Fludarabine plus 2 Gy total body irradiation $(n=10)$. In this regimen, patients receive identical tolerized T cell doses and graft vs. host disease prophylaxis. Lymphocyte polarization is avoided as donors begin growth factors after lymphocyte collection.

on a two step HI HSCT study was included in this analysis if they met these criteria. Patients had lymphoid or myeloid malignancies. Five patients with aplastic anemia were included in the univariable analyses only for GVHD and OS.

\section{Statistical Analysis}

Comparison of groups was performed using Fisher's exact test for categorical variables and ANOVA for continuous variables. For the outcomes analysis, univariate association of recipient characteristics with endpoints was evaluated using the competing risk method of Gray (46) (for relapse, NRM, aGVHD, and cGVHD) or the log rank test (OS). Analysis of aGVHD was censored at 180 days. Multivariable competing risk proportional hazards regression used the method of Fine and Gray (47). Cox proportional hazards regression was used for multivariable models of OS. NRM was the competing event for relapse, relapse was the competing event for NRM, and death was the competing event for aGVHD and cGVHD. Each endpoint was evaluated separately in CMV+ and CMVrecipients. Multivariable models assessed the association of CMV IMD allele with each outcome adjusted for recipient and donor age, paternal recipient, RDRI (categorized as High vs. Low), diagnosis, conditioning regimen, and race. Race was analyzed as a three category variable (Caucasian/African American/Other Minority) with type $3 p$-values reported for this variable. Models for relapse, NRM, cGVHD and OS were also adjusted for steroid administration within 100 days and $\mathrm{CMV}$ reactivation within 100 days $(\mathrm{CMV}+$ sample only). All analyses were completed using SAS version 9.4 (SAS Institute, Cary, NC).

\section{Definitions}

CMV reactivation was defined as a positive result of $>100$ $\mathrm{IU} / \mathrm{ml}$ on quantitative, real-time PCR testing of plasma within 100 days of HSCT. Preemptive therapy with valganciclovir was instituted for CMV reactivation. CMV IMD alleles were identified as HLA $A^{*} 01: 01, A^{*} 02: 01, B^{*} 07: 02, B^{*} 08: 01$, and $\mathrm{C}^{*}$ 07:02. aGVHD (grades 2-4) and CGVHD were identified and scored based on Glucksberg et al. (48) and National Institutes of Health Consensus Criteria, respectively (49). Relapse was defined as morphologic recurrence of disease. NRM was defined as death from any cause while the underlying malignancy was in remission. AML, myelodysplastic syndrome, chronic myelomonocytic leukemia, and myeloproliferative disorder were defined as myeloid malignancies.

\section{RESULTS}

\section{Patients}

The outcomes of 216 consecutive two-step patients meeting inclusion criteria were analyzed. Supplementary Tables 1, 2 contain complete details of the analyses. There were 127 $\mathrm{CMV}+$ and $89 \mathrm{CMV}$ - recipients. Median follow-up of the participants was 23.7 months. Median and mean peripheral blood donor $\mathrm{T}$ cell chimerism of the group was 100 and $99.3 \%$, respectively. Patient characteristics and association of confounders with CMV IMD alleles are contained in Table 1. Included in the analysis were 150 Caucasians, 45 African Americans, and 21 patients characterized as "Other Minority” (Non-African America minorities: 9 Hispanic, 11 Asian, and 1 Multiple Race). As expected, Caucasians possessed a significantly higher percentage of CMV IMD alleles compared to non-Caucasians in both the $\mathrm{CMV}+$ and CMV- groups.

\section{Serostatus, Reactivation, and Tissue Disease}

In the total healthy donor population of 216 subjects, the presence of a CMV IMD allele predicted for a CMV negative serostatus. Fifty-two of 136 (38\%) CMV IMD allele+ donors vs. 46/80 (58\%) CMV IMD allele- donors were $\mathrm{CMV}+$, Chi Square 0.007. The same trend occurred in the recipient group where $75 / 138(54 \%)$ of CMV IMD allele+ vs. $52 / 78(67 \%)$ of CMV IMD- recipients were CMV+ although these differences did not reach statistical significance, Chi Square $p=0.077$.

In the CMV+ group, 101/127 (80\%) of patients had laboratory evidence of CMV reactivation within the first 100 days of $\mathrm{HI}$ HSCT. There was no significant difference in CMV reactivation rates based on the presence or absence of a recipient CMV IMD allele, 80 vs. $79 \%$, respectively, Chi-Square $p=0.87$. 
TABLE 1 | Associations of confounders with CMV IMD alleles.

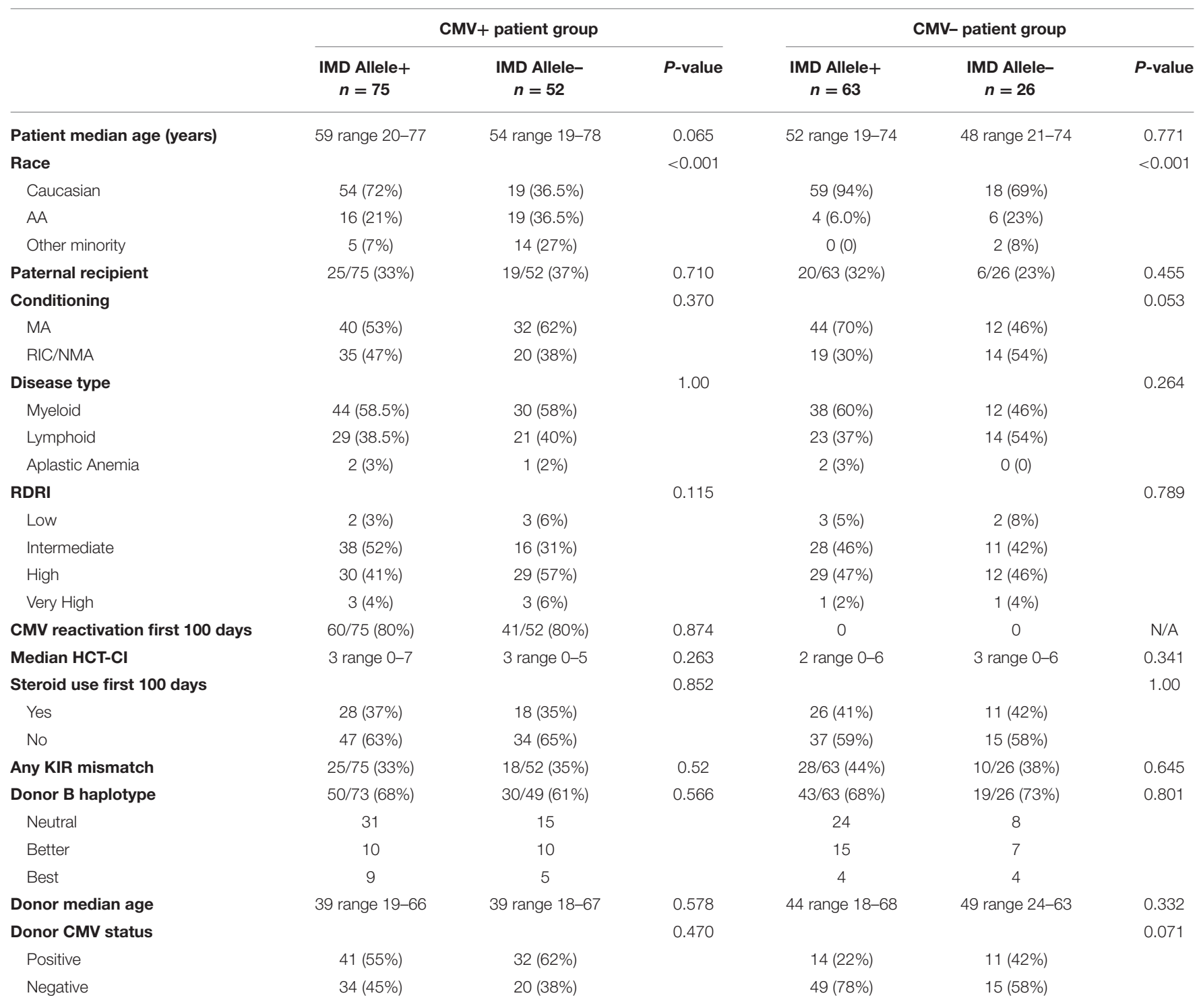

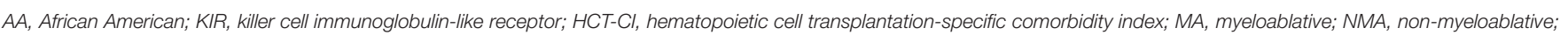
$R D R I$, revised disease risk index; $R I C$, reduced intensity conditioning.

Nine of 127 (7\%) patients in the CMV+ group developed tissue manifestations of CMV. In this group of 9 patients, $2 / 3$ of patients were CMV IMD allele-, and $2 / 3$ of patients had CMVdonors. In five patients, CMV tissue disease developed in the gut (4) and retina (1) between days 95 and 365 (median $d+170$ ) in the context of treatment with steroids for GVHD. In the remaining 4 patients, CMV tissue disease developed earlier in the gut (2) and lung (2), between days 33 and 52 (median d +48 ). All four of these earlier patients were CMV IMD alleleand 2/4 had CMV- donors. Only one of these four early patients was being treated for GVHD with steroids at the time of tissue disease. CMV pneumonitis was the primary cause of death in two patients-both in the early group. These patients were not being treated for GVHD at the time of tissue disease, were CMV IMD allele-, and one of the two patients had a CMV-donor.
CMV tissue disease was treated with foscarnet or ganciclovir, and in the cases of CMV pneumonitis, CMV-specific gamma globulin.

No patient in the CMV- group had evidence of primary CMV infection post HSCT.

\section{Relapse}

In CMV + recipients, the presence of a CMV IMD allele was associated with a lower hazard of relapse $(\mathrm{HR}=0.47 p=0.136)$ although in the current analysis, the result did not reach statistical significance. Cumulative incidence (CI) plot (Figure 2) shows that $\mathrm{CMV}+$ recipients who lacked a CMV IMD allele had the highest relapse rate amongst the $\mathrm{CMV}+$ and $\mathrm{CMV}-$ groups although this analysis also failed to reach statistical significance $(p=0.213)$. CI relapse in CMV+/IMD allele+ vs. CMV+/IMD 


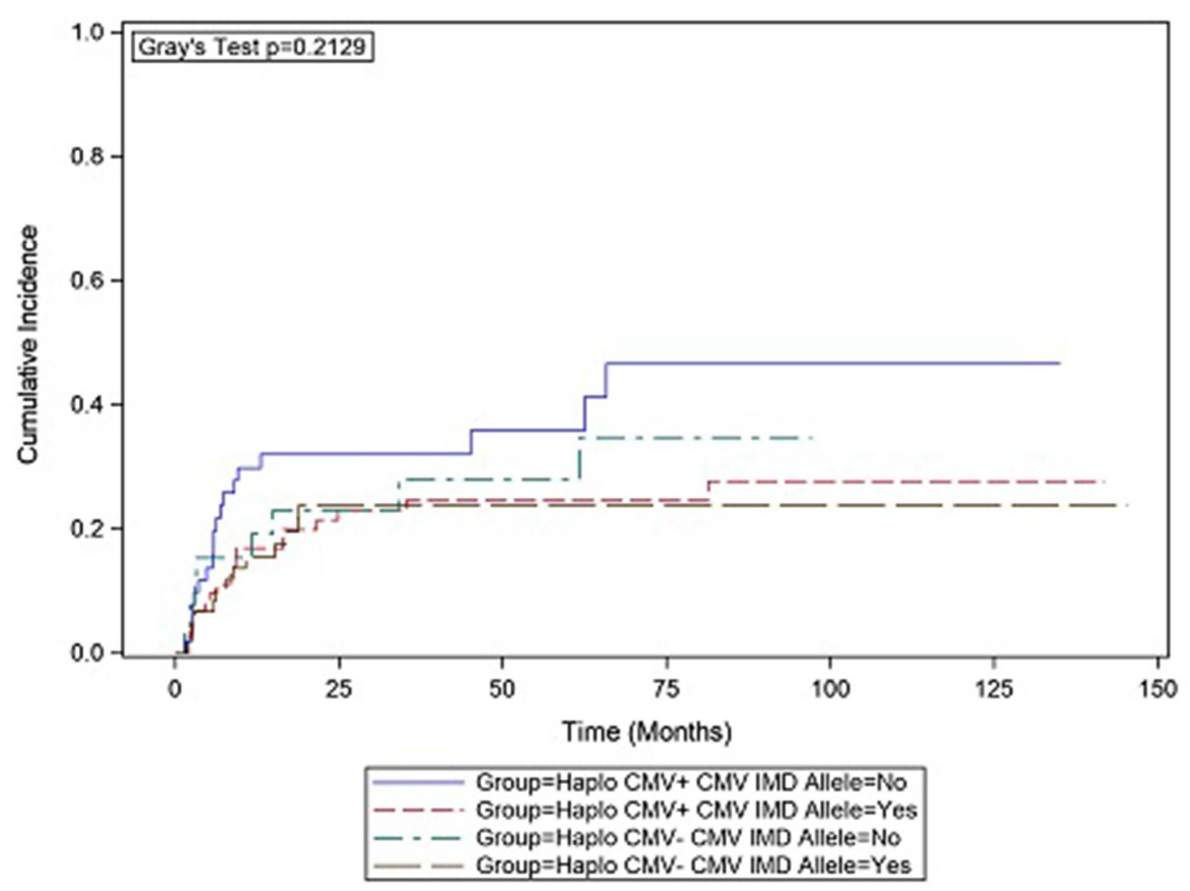

FIGURE 2 | CMV+ patients without a CMV immunodominant allele had the highest cumulative incidence of relapse. although the results did not reach statistical significance.

allele- patients at 2 years was 23 vs. $32 \%$, respectively. In the $\mathrm{CMV}+$ group, a low RDRI score $(\mathrm{HR}=0.44, p=0.03)$ and paternal recipient status $(\mathrm{HR}=0.25, p=0.005)$ were associated with lower relapse rates.

\section{Acute GVHD}

The presence or absence of a CMV IMD allele was not associated with aGVHD in either CMV+ or CMV- recipients. While not reaching statistical significance $(p=0.123)$, Caucasians were more likely to develop aGVHD in both the CMV+ and CMVgroups $(\mathrm{HR}=2.13$ and 3.42 , respectively) as compared to African Americans.

\section{Chronic GVHD}

cGVHD was not related to the presence or absence of a CMV IMD allele in $\mathrm{CMV}+$ or $\mathrm{CMV}-$ recipients. In the $\mathrm{CMV}+$ group, father recipient ( $\mathrm{HR}=3.57, p=0.046)$ was associated with the development of cGVHD, while a high RDRI score was associated with a decreased hazard of cGVHD (HR = $0.20, p=0.012$ ). Race was associated with cGVHD (Type 3 $p=0.003)$ in the CMV+ group. Caucasians were less likely to develop cGVHD ( $\mathrm{HR}=0.201, p=0.0078)$ and nonAfrican American minorities had a relatively equal hazard of developing cGVHD ( $\mathrm{HR}=1.2, p=0.82)$ as compared to African Americans. The CMV- group analysis for the impact of race on cGVHD was not able to be assessed due to the low number of non-African American minorities in this cohort.

\section{Non-relapse Mortality}

The presence or absence of a CMV IMD allele was not significantly associated with NRM in any group. In CMV+ recipients, HR for NRM was 0.73 in CMV IMD allele+ vs. 1.37 in CMV IMD allele- recipients $(p=0.43)$. In CMV- recipients HR for NRM was 0.47 in CMV IMD allele+ vs. 2.137 in CMV IMD allele- recipients $(p=0.13)$ In $\mathrm{CMV}+$ recipients, CMV reactivation $(\mathrm{HR}=7.27, p=0.057)$ and the use of steroids in the first 100 days ( $\mathrm{HR}=2.87, p=0.015)$ were associated with an increase in NRM. Race was not associated with NRM.

\section{Survival}

CMV reactivation was associated with increased mortality (HR $=3.42, p=0.007)$. However, the risk of death was considerably lower in $\mathrm{CMV}+$ recipients possessing a CMV IMD allele $(\mathrm{HR}=$ $0.40, p=0.003)$ vs. those $\mathrm{CMV}+$ recipients in which an allele was not present. As shown in the CI plot (Figure 3), the presence of a CMV IMD allele in CMV+ patients greatly increased OS compared to $\mathrm{CMV}+$ recipients without the allele (Logrank $p=$ 0.0035). CI of OS in CMV+/IMD allele+ vs. CMV+/IMD allelepatients at 2 years was 63 vs. $41 \%$, respectively. CMV+ patients possessing a CMV IMD allele experienced OS curves that were similar to their CMV- counterparts. The presence of a CMV IMD allele was also associated with a lower hazard of death in CMV- recipients $(\mathrm{HR}=0.58)$, although the result did not reach statistical significance $(p=0.18)$.

In both groups, mortality was higher with increasing RDRI score reaching significance in the $\mathrm{CMV}+$ group $(\mathrm{HR}=1.97, p$ $=0.015)$. Risk of death was lower in recipients with myeloid 


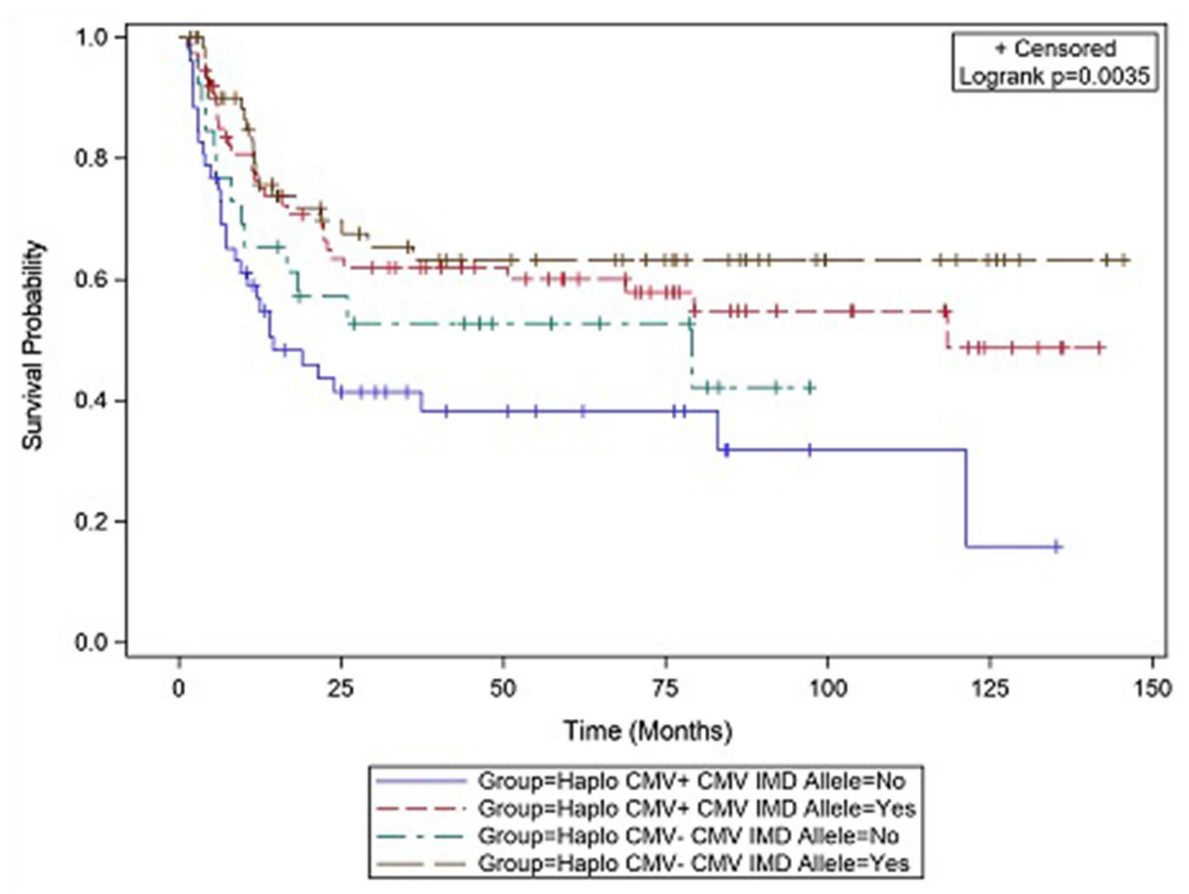

FIGURE 3 | Survival differences in CMV+ vs. CMV- patients based on the presence or absence of a CMV immunodominant allele.

vs. lymphoid diseases, reaching statistical significance in the $\mathrm{CMV}+$ group $(\mathrm{HR}=0.45, p=0.011)$. There were no significant differences in risk of mortality based on race/ethnicity; Caucasian $(\mathrm{HR}=0.92)$ and non-Caucasian non-African American $(\mathrm{HR}=$ 0.91 , type $3 p=0.956$ ) vs. African American recipients. CMV+ Caucasian recipients not in possession of a CMV IMD allele had a significantly lower OS rate vs. CMV + Caucasians with a CMV IMD allele on Kaplan Meier analysis, $p=0.024$ (Figure 4).

\section{DISCUSSION}

The primary finding of this multivariable analysis was that the presence of a CMV IMD allele in CMV + recipients undergoing HI HSCT significantly reduced the hazard of post-transplant death as compared to those not possessing a CMV IMD allele. Moreover, the possession of a CMV IMD allele served to increase the OS rate in CMV+ recipients, most of whom developed CMV reactivation, to that of $\mathrm{CMV}$ - recipients none of whom had evidence of CMV post HSCT. Accordingly, CMV+ recipients without a CMV IMD allele had the poorest CI of OS at $<20 \%$.

In contrast to our preliminary univariable analysis (38), the impact of CMV IMD alleles on relapse incidence and NRM in $\mathrm{CMV}+$ recipients was not statistically significant in this multivariable study. Because the hazards for NRM and especially for relapse were lower in the CMV IMD allele+ group, it is possible that a combination effect from these factors was responsible for the OS benefit. However, CMV reactivation was significantly associated with increased NRM in CMV+ recipients, and reactivation occurred equally between CMV IMD allele+ and CMV IMD allele- groups. This finding suggests that the OS benefit associated with the possession of a CMV IMD allele was derived more substantively from relapse protection.

The preliminary analysis (38) as well as an internal multivariable analysis (Grosso, unpublished) showed that any benefits derived from the presence CMV IMD alleles were specific to the possession of one or more of these alleles by the recipient only. Responding CTLs in this analysis were of donor origin suggesting that the effects were due to recipient antigen presentation.

The ability to compensate for CMV immunoevasions $(27,50)$ and to present multiple CMV epitopes $(8,24)$ are characteristics of the CMV IMD alleles tested in this analysis. More efficient antigen presentation by host antigen presenting cells expressing a CMV IMD dominant allele or their ability to stimulate higher frequency donor CMV-specific CTL responses potentially strengthened a graft vs. tumor response resulting in a survival benefit. Testing of this hypothesis and the results of this analysis in general require further study.

There was no evidence that the presence of a CMV IMD allele was related to the occurrence of GVHD, nor was there evidence that the presence of a recipient CMV IMD allele affected HSCT outcomes, including OS, in the absence of CMV reactivation.

In some HI HSCT settings, natural killer (NK) effects such as KIR mismatch between the donor and the recipient or the presence of an activating KIR centromeric and telomeric B motif in the donor, are associated with lower relapse rates and improved OS aft $\operatorname{HSCT}(42,43)$. Because there was an equal distribution of these characteristics between the CMV IMD positive and negative groups, we do not believe that OS differences in this analysis were due to NK effects. 


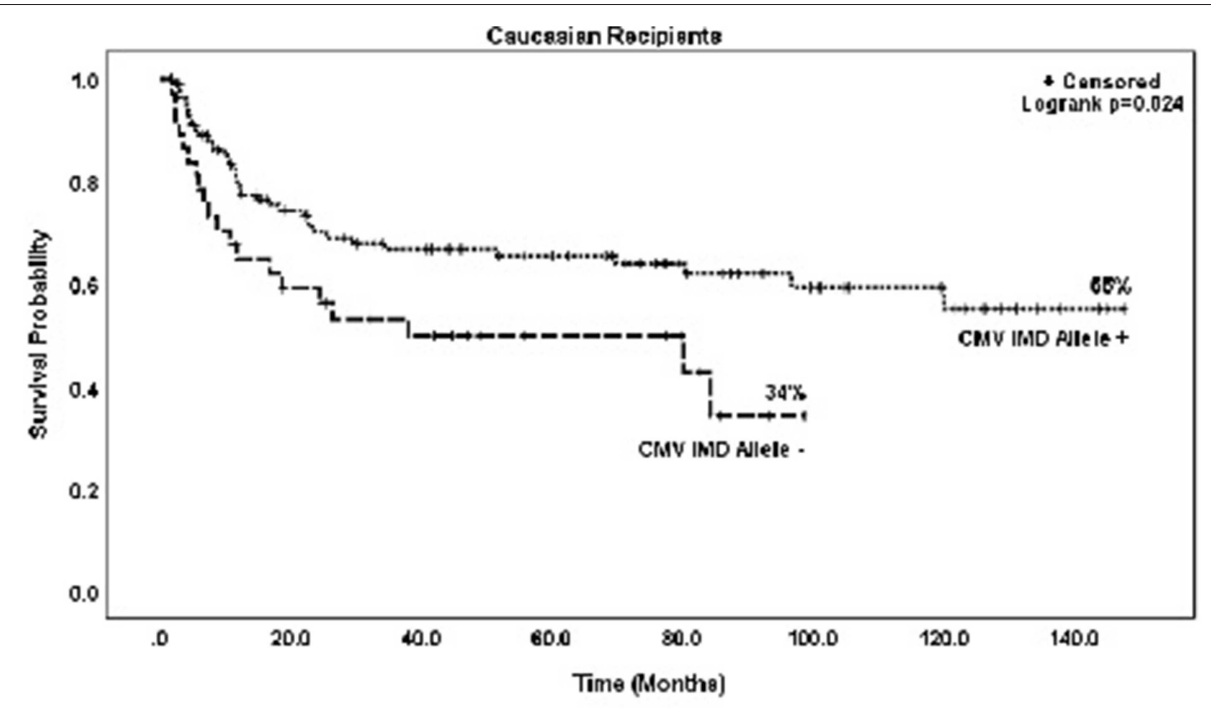

FIGURE 4 | Probability of survival in CMV+ Caucasian patients based on the presence or absence of a CMV immunodominant allele.

In contrast to the strong association between CMV IMD alleles and improved survival, mortality differences between different races and ethnicities was not observed. This finding is inconsistent with CMV IMD alleles serving as surrogates for Caucasian race in this analysis. The finding that Caucasians not possessing CMV IMD alleles had lower OS rates than Caucasians with these alleles strengthens this conclusion. There was a highly significant relationship between non-Caucasians and an increased incidence of cGVHD in the current study. This finding is in agreement with Solomon et al. (51) in which African Americans undergoing post-transplant CY HI HSCT were found to have higher incidences of cGVHD, but in contrast to our analysis, lower rates of mortality and relapse as compared to Caucasians. There is limited information regarding the effect of race on outcomes post HI HSCT in general and this area requires continued investigation.

We opted to include the small percentage of $\mathrm{CMV}+$ patients who had no microbiologic evidence of CMV reactivation post HI HSCT in the CMV+ group to avoid the loss of subjects with undetected, transient CMV reactivation. We have demonstrated large increases in CTL frequency after CMV reactivation in $\mathrm{HI}$ HSCT recipients $(22,52,53)$. While the median $d+90 \mathrm{CD} 3 / 8^{+}$ cell frequency of these CMV+ "non-reactivators" was lower than that of CMV+ patients reactivating CMV (Mann-Whitney $p$ $=0.005$ ), it was significantly higher than patients in the CMVgroup (Mann-Whitney $p=0.019$ ). This suggested that there was likely subclinical, undetected CMV reactivation in at least some subjects who were classified as not reactivating CMV justifying inclusion into CMV+ testing group.

Limitations of this study include its retrospective nature and specificity to the two step population. While the homogeneous nature of the two step HI HSCT approach reduces the confounders of varying $\mathrm{T}$ cell doses and GVHD prophylaxis strategies on the current results, a broader analyses of patient outcomes based on the presence or absence of CMV IMD alleles in the setting of alternate HI-HSCT approaches should be performed to confirm these results. In vitro studies specifically examining anti-CMV responses and potential anti-tumor effects of cross-reactive CMV-specific CTLs in patients possessing $\mathrm{CMV}$ alleles of varying immunodominance are planned at our institution to further explore these results.

In summary, the presence of a CMV IMD allele as defined in this study, was associated with protection from death in patients reactivating CMV after HI HSCT. To our knowledge, this is the first analysis of post HI HSCT outcomes based on the presence or absence of a CMV IMD allele. The data potentially delineates a high risk subpopulation of patients within the CMV+ group who do not benefit from a GVT effect related to CMV reactivation while simultaneously at risk for the negative effects of CMV reactivation. Consequently, the CMV IMD allelegroup within the CMV+ population is the most likely to benefit from CMV prophylaxis with DNA terminase complex inhibitors. Another question raised by the data is whether the OS benefit associated with CMV reactivation in the CMV+/CMV IMD allele+ population justifies the withholding of DNA terminase complex inhibitors in this group. Additional analyses in larger groups of patients to assess whether the potential beneficial effects of CMV reactivation on both relapse and NRM outweigh deleterious effects in the CMV IMD allele+ subgroup. The results of this study support continued analysis in this area.

\section{DATA AVAILABILITY}

The datasets generated for this study are available on request to the corresponding author.

\section{ETHICS STATEMENT}

This studies involving human participants were reviewed and approved by Thomas Jefferson University Institutional Review 
Board. The patients/participants provided their written informed consent to participate in this study.

\section{AUTHOR CONTRIBUTIONS}

DG developed the research, interpreted the analysis, and was the primary manuscript author. BL performed the statistical analysis and assisted in interpreting the data. MC, JF-O'H, SG, WO' $\mathrm{H}$, and JW assisted in the interpretation of the data, contributed

\section{REFERENCES}

1. Green ML, Leisenring WM, Xie H, Walter RB, Mielcarek M, Sandmaier $\mathrm{BM}$, et al. CMV reactivation after allogeneic HCT and relapse risk: evidence for early protection in acute myeloid leukemia. Blood. (2013) 122:1316-24. doi: 10.1182/blood-2013-02-487074

2. Manjappa S, Bhamidipati PK, Stokerl-Goldstein KE, DiPersio JF, Uy GL, Westervelt $\mathrm{P}$, et al. Protective effect of cytomegalovirus reactivation on relapse after allogeneic hematopoietic cell transplantation in acute myeloid leukemia patients is influenced by conditioning regimen. Biol Blood Marrow Transplant. (2014) 20:46-52. doi: 10.1016/j.bbmt.2013.10.003

3. Litjens NHR, van der Wagen L, Kuball J, Kwekkeboom J. Potential beneficial effects of cytomegalovirus infection after transplantation. Front Immunol. (2018) 9:389. doi: 10.3389/fimmu.2018.00389

4. Elmaagacli AH, Koldehoff M. Cytomegalovirus replication reduces the relapse incidence in patients with acute myeloid leukemia. Blood. (2016) 128:456. doi: 10.1182/blood-2016-04-713644

5. Takenaka K, Nishida T, Asano-Mori Y, Oshima K, Ohashi K, Mori T, et al. Cytomegalovirus reactivation after allogeneic hematopoietic stem cell transplantation is associated with a reduced risk of relapse in patients with acute myeloid leukemia who survived to day 100 after transplantation: The Japan Society for hematopoietic cell transplantation transplantation-related complication working group. Biol Blood Marrow Transplant. (2015) 21:200816. doi: 10.1016/j.bbmt.2015.07.019

6. Teira P, Battiwalla M, Ramanathan M, Barrett AJ, Ahn KW, Chen M, et al. Early cytomegalovirus reactivation remains associated with increased transplant-related mortality in the current era: a CIBMTR analysis. Blood. (2016) 127:2427-38. doi: 10.1182/blood-2015-11-679639

7. Hall CE, Koparde VN, Jameson-Lee M, Elnasseh AG, Scalora AF, Kobulnicky DJ, et al. Sequence homology between HLA-bound cytomegalovirus and human peptides: a potential trigger for alloreactivity. PLoS ONE. (2017) 12:e0178763. doi: 10.1371/journal.pone.0178763

8. Louzoun Y, Vider T, Weigert M. T-cell epitope repertoire as predicted from human and viral genomes. Mol Immunol. (2006) 43:559-69. doi: 10.1016/j.molimm.2005.04.017

9. Hertz T, Nolan D, James I, John M, Gaudieri S, Phillips E, et al. Mapping the landscape of host-pathogen coevolution: HLA class I binding and its relationship with evolutionary conservation in human and viral proteins. $J$ Virol. (2011) 85:1310-21. doi: 10.1128/JVI.01966-10

10. Gaballa S, Palmisiano N, Alpdogan O, Carabasi M, Filicko-O’Hara J, Kasner M, et al. A two-step haploidentical versus a two-step matched related allogeneic myeloablative peripheral blood stem cell transplantation. Biol Blood Marrow Transplant. (2016) 22:141-8. doi: 10.1016/j.bbmt.2015. 09.017

11. Hebert C, Watts N, Isaac S, Kukkamalla R, Jamy O, Saad A. Cytomegalovirus reactivation after allogeneic hematopoietic stem cell transplantation with post-transplant cyclophosphamide. Biol Blood Marrow Transplant. (2017) 23:S276-7. doi: 10.1016/j.bbmt.2016.12.183

12. Al Malki MM, Dadwal S, Yang D, Mokhtari S, Cao T, Gendzekhadze K. High incidence of CMV reactivation after haploidentical donor hematopoietic cell transplantation using high-dose post-transplant cyclophosphamide, and its impact on transplant outcomes. Blood. (2017) 130:4494.

13. Wang TP, Sochat M, Tan CRC, Hossain NM, Ulicny J, Pancari PA, et al. $\mathrm{CMV}$ and $\mathrm{BK}$ virus reactivation in post transplant cyclophosphamide (PTCY) to, and edited the manuscript. NF assisted in developing the research, analyzing results, and editing and approving of the manuscript.

\section{SUPPLEMENTARY MATERIAL}

The Supplementary Material for this article can be found online at: https://www.frontiersin.org/articles/10.3389/fonc. 2019.00888/full\#supplementary-material

after allogeneic MUD, MRD and haploidentical transplant. Biol Blood Marrow Transplant. (2017) 23:S305-6. doi: 10.1016/j.bbmt.2016.12.227

14. Lin $\mathrm{CH}, \mathrm{Su} \mathrm{YJ}$, Hsu CY, Wang PN, Teng CJ. Haploidentical allogeneic hematopoietic stem cell transplantation increases the risk of cytomegalovirus infection in adult patients with acute leukemia. Transplant Infect Dis. (2019) 21:e13096. doi: 10.1111/tid.13096

15. Yokoyama H, Kanda J, Kato S, Kondo E, Maeda Y, Saji H, et al. Effects of HLA mismatch on cytomegalovirus reactivation in cord blood transplantation. Bone Marrow Transplant. (2019) 54:1004-12. doi: 10.1038/s41409-018-0369-0

16. Liu J, Chang YJ, Yan CH, Xu LP, Jiang ZF, Zhang XH, et al. Poor CMV-specific $\mathrm{CD}^{+} \mathrm{T}$ central memory subset recovery at early stage post-HSCT associates with refractory and recurrent CMV reactivation. J Infect. (2016) 73:261-70. doi: $10.1016 /$ j.jinf.2016.04.033

17. Pei XY, Zhao XY, Chang YJ, Liu J, Xu LP, Wang Y, et al. Cytomegalovirusspecific T-cell transfer for refractory cytomegalovirus infection after haploidentical stem cell transplantation: the quantitative and qualitative immune recovery for cytomegalovirus. J Infect Dis. (2017) 216:945-56. doi: 10.1093/infdis/jix357

18. Hammerstrom AE, Lombardi LR, Pingali SR, Rondon G, Chen J, Milton $\mathrm{DR}$, et al. Prevention of cytomegalovirus reactivation in haploidentical stem cell transplantation. Biol Blood Marrow Transplant. (2018) 24:353-8. doi: 10.1016/j.bbmt.2017.09.018

19. Lin $\mathrm{X}$, Ou Y, Long $\mathrm{H}$, Huang $\mathrm{Y}$, Song $\mathrm{C}, \mathrm{Lu} \mathrm{Z}$, et al. Cytomegalovirus infection after haploidentical stem cell transplantation may reduce relapse risk in leukemia. Zhonghua Nei Ke Za Zhi. (2016) 55:107-10. doi: 10.3760/cma.j.issn.0578-1426.2016.02.008

20. Dioverti MV, Huang C, Morales M, Lu N, Cochran W, Gladstone D, et al. Cytomegalovirus reactivation in haploidentical blood or marrow transplant (BMT) Using post-transplant cyclophosphamide (PTCy) is associated with higher non-relapse-related mortality, despite rarity of disease. Blood. (2018) 132:2096. doi: 10.1182/blood-2018-99-119603

21. Goldsmith SR, Slade M, Lawrence SJ, DiPersio JF, Westervelt P, Uy GL, et al. Cytomegalovirus viremia and relapse after haploidentical hematopoietic cell transplantation. Biol Blood Marrow Transplant. (2016) 22:S320. doi: 10.1016/j.bbmt.2015.11.795

22. Gooptu M, Leiby B, Alpdogan O, Carabasi M, Filicko-O’Hara J, Kasner M. CD3/8 T-cell responses to CMV reactivation in patients undergoing haploidentical transplants: a retrospective analysis. Blood. (2014) 124:1170. doi: 10.1016/j.bbmt.2014.11.241

23. Kondo E, Akatsuka Y, Kuzushima K, Tsujimura K, Asakura S, Tajima K, et al. Identification of novel CTL epitopes of CMV-pp65 presented by a variety of HLA alleles. Blood. (2003) 103:630. doi: 10.1182/blood-2003-03-0824

24. Hyun SJ, Sohn HJ, Lee HJ, Lee SD, Kim S, Sohn DH, et al. Comprehensive analysis of Cytomegalovirus pp65 antigen-specific CD8(+) T cell responses according to human leukocyte antigen class I allotypes and intraindividual dominance. Front Immunol. (2017) 8:1591. doi: 10.3389/fimmu.2017.01591

25. Lidehall AK, Sund F, Lundberg T, Eriksson BM, Tötterman TH, Korsgren O. $\mathrm{T}$ cell control of primary and latent cytomegalovirus infections in healthy subjects. J Clin Immunol. (2005) 25:473-81. doi: 10.1007/s10875-005-5372-8

26. Morita Y, Hosokawa M, Ebisawa M, Sugita T, Miura O, Takaue Y, et al. Evaluation of cytomegalovirus-specific cytotoxic T-lymphocytes in patients with the HLA-A*02 or HLA-A*24 phenotype undergoing hematopoietic stem cell transplantation. Bone Marrow Transplant. (2005) 36:803. doi: $10.1038 /$ sj.bmt. 1705133 
27. Ameres S, Mautner J, Schlott F, Neuenhahn M, Busch DH, Plachter B, et al. Presentation of an immunodominant immediate-early CD8 $+\mathrm{T}$ cell epitope resists human cytomegalovirus immunoevasion. PLoS Pathog. (2013) 9:e1003383. doi: 10.1371/journal.ppat.1003383

28. Khan N, Best D, Bruton R, Nayak L, Rickinson AB, Moss PA. T cell recognition patterns of immunodominant cytomegalovirus antigens in primary and persistent infection. J Immunol. (2007) 178:4455. doi: 10.4049/jimmunol.178.7.4455

29. Kern F, Faulhaber N, Frömmel C, Khatamzas E, Prösch S, Schönemann $\mathrm{C}$, et al. Analysis of CD8 T cell reactivity to cytomegalovirus using protein-spanning pools of overlapping pentadecapeptides. Eur J Immunol. (2000) 30:1676-82. doi: 10.1002/1521-4141(200006)30:6<1676::AID-IMMU1676>3.0.CO;2-V

30. Hosie L, Pachnio A, Zuo J, Pearce H, Riddell S, Moss P. Cytomegalovirusspecific $\mathrm{T}$ cells restricted by HLA-Cw*0702 increase markedly with age and dominate the $\mathrm{CD}^{+}$T-cell repertoire in older people. Front Immunol. (2017) 8:1776. doi: 10.3389/fimmu.2017.01776

31. Lacey SF, Villacres MC, La Rosa C, Wang Z, Longmate J, Martinez J, et al. Relative dominance of HLA-B*07 restricted $\mathrm{CD}^{+}$T-lymphocyte immune responses to human cytomegalovirus pp65 in persons sharing HLA-A*02 and HLA-B*07 alleles. Hum Immunol. (2003) 64:440-52. doi: 10.1016/S0198-8859(03)00028-4

32. Braendstrup P, Mortensen BK, Justesen S, Osterby T, Rasmussen M, Hansen $\mathrm{AM}$, et al. Identification and HLA-tetramer-validation of human $\mathrm{CD}^{+}$and $\mathrm{CD}^{+} \mathrm{T}$ cell responses against HCMV proteins IE1 and IE2. PLOS ONE. (2014) 9:e94892. doi: 10.1371/journal.pone.0094892

33. Schlott F, Steubl D, Ameres S, Moosmann A, Dreher S, Heemann U, et al. Characterization and clinical enrichment of HLA-C*07:02-restricted Cytomegalovirus-specific CD8+ T cells. PLOS ONE. (2018) 13:e0193554. doi: 10.1371/journal.pone.0193554

34. Giest S, Grace S, Senegaglia AC, Pasquini R, Gonzalo-Daganzo RM, Fernández $\mathrm{MN}$, et al. Cytomegalovirus-specific $\mathrm{CD}^{+} \mathrm{T}$ cells targeting different HLA/peptide combinations correlate with protection but at different threshold frequencies. $\mathrm{Br}$ J Haematol. (2010) 148:311-22. doi: 10.1111/j.1365-2141.2009.07969.x

35. Wills MR, Carmichael AJ, Mynard K, Jin X, Weekes MP, Plachter B, et al. The human cytotoxic T-lymphocyte (CTL) response to cytomegalovirus is dominated by structural protein pp65: frequency, specificity, and T-cell receptor usage of pp65-specific CTL. J Virol. (1996) 70:7569-79.

36. Elkington R, Walker S, Crough T, Menzies M, Tellam J, Bharadwaj M, et al. Ex vivo profiling of $\mathrm{CD} 8(+)$-T-cell responses to human cytomegalovirus reveals broad and multispecific reactivities in healthy virus carriers. J Virol. (2003) 77:5226-40. doi: 10.1128/JVI.77.9.5226-5240.2003

37. Ogonek J, Verma K, Schultze-Florey C, Varanasi P, Luther S, Schweier $\mathrm{P}$, et al. Characterization of high-avidity cytomegalovirusspecific $t$ cells with differential tetramer binding coappearing after allogeneic stem cell transplantation. J Immunol. (2017) 199:792-805. doi: 10.4049/jimmunol.1601992

38. Grosso D, Alpdogan O, Carabasi M, Filicko-O’Hara J, Gaballa S, Kasner M, et al. The presence of recipient CMV immunodominant human leukocyte antigen (HLA) alleles affect outcomes after haploidentical (HI) hematopoietic stem cell transplantation (HSCT). Blood. (2017) 130:1959.

39. Geethakumari PR, Leiby B, Nair R, Alpdogan SO, Carabasi M, FilickoO'Hara J, et al. Higher rates of relapse in maternal recipients of haploidentical hematopoietic stem cell transplantation from adult offspring donors for AML and myelodysplastic syndrome. Bone Marrow Transplant. (2017) 52:1465-7. doi: 10.1038/bmt.2017.159

40. Armand P, Kim HT, Logan BR, Wang Z, Alyea EP, Kalaycio ME, et al. Validation and refinement of the disease risk index for allogeneic stem cell transplantation. Blood. (2014) 123:3664-71. doi: 10.1182/blood-2014-01-552984
41. Sorror ML, Maris MB, Storb R, Baron F, Sandmaier BM, Maloney DG, et al. Hematopoietic cell transplantation (HCT)-specific comorbidity index: a new tool for risk assessment before allogeneic HCT. Blood. (2005) 106:2912-9. doi: 10.1182/blood-2005-05-2004

42. Ruggeri L, Capanni M, Urbani E, Perruccio K, Shlomchik WD, Tosti A, et al. Effectiveness of donor natural killer cell alloreactivity in mismatched hematopoietic transplants. Science. (2002) 295:2097-100. doi: 10.1126/science.1068440

43. Cooley S, Weisdorf DJ, Guethlein LA, Klein JP, Wang T, Le CT, et al. Donor selection for natural killer cell receptor genes leads to superior survival after unrelated transplantation for acute myelogenous leukemia. Blood. (2010) 116:2411-9. doi: 10.1182/blood-2010-05-283051

44. Baker KS, Davies SM, Majhail NS, Hassebroek A, Klein JP, Ballen KK, et al. Race and socioeconomic status influence outcomes of unrelated donor hematopoietic cell transplantation. Biol Blood Marrow Transplant. (2009) 15:1543-54. doi: 10.1016/j.bbmt.2009.07.023

45. Mielcarek M, Gooley T, Martin PJ, Chauncey TR, Young BA, Storb R, et al. Effects of race on survival after stem cell transplantation. Biol Blood Marrow Transplant. (2005) 11:231-9. doi: 10.1016/j.bbmt.2004.12.327

46. Gray RJ. A class of K-sample tests for comparing the cumulative incidence of a competing risk. Ann Statist. (1988) 16:1141-54. doi: 10.1214/aos/1176350951

47. Fine JP, Gray RJ. A proportional hazards model for the subdistribution of a competing risk. J Am Stat Assoc. (1999) 94:496-509. doi: 10.1080/01621459.1999.10474144

48. Glucksberg H, Storb R, Fefer A, Buckner CD, Neiman PE, Clift RA, et al. Clinical manifestations of graft-versus-host disease in human recipients of marrow from HL-A-matched sibling donors. Transplantation. (1974) 18:295304. doi: 10.1097/00007890-197410000-00001

49. Filipovich AH, Weisdorf D, Pavletic S, Socie G, Wingard JR, Lee SJ, et al. National institutes of health consensus development project on criteria for clinical trials in chronic graft-versus-host disease: I. Diagnosis and staging working group report. Biol Blood Marrow Transplant. (2005) 11:945-56. doi: 10.1016/j.bbmt.2005.09.004

50. Holtappels R, Thomas D, Reddehase MJ. The efficacy of antigen processing is critical for protection against cytomegalovirus disease in the presence of viral immune evasion proteins. J Virol. (2009) 83:9611. doi: 10.1128/JVI. 00936-09

51. Solomon SR, Zhang X, Holland HK, Morris LE, Solh M, Bashey A. Superior survival of black versus white patients following post-transplant cyclophosphamide-based haploidentical transplantation for adults with hematologic malignancy. Biol Blood Marrow Transplant. (2018) 24:1237-42. doi: 10.1016/j.bbmt.2018.01.024

52. Anikeeva N, Grosso D, Flomenberg N, Sykulev Y. Evaluating frequency and quality of pathogen-specific T cells. Nat Commun. (2016) 7:13264. doi: $10.1038 /$ ncomms 13264

53. Grosso D, Anikeeva N, Sylulev Y, Flomenberg N. CMV responses after haploidentical hematopoietic stem cell transplantation (HI HSCT): quantity does not always equal quality. Blood. (2018) 132:3372. doi: 10.1182/blood-2018-99-115265

Conflict of Interest Statement: The authors declare that the research was conducted in the absence of any commercial or financial relationships that could be construed as a potential conflict of interest.

Copyright (C) 2019 Grosso, Leiby, Carabasi, Filicko-O'Hara, Gaballa, O'Hara, Wagner and Flomenberg. This is an open-access article distributed under the terms of the Creative Commons Attribution License (CC BY). The use, distribution or reproduction in other forums is permitted, provided the original author(s) and the copyright owner(s) are credited and that the original publication in this journal is cited, in accordance with accepted academic practice. No use, distribution or reproduction is permitted which does not comply with these terms. 